\title{
A LINEAR PARABOLIC BOUNDARY CONTROL PROBLEM WITH MIXED CONTROL-STATE CONSTRAINT
}

Maïtine Bergounioux

Département de Mathématiques Faculty of Mathematics UMR 6628 -

Université d'Orléans, France

\section{Fredi Tröltzsch}

Fakultät für Mathematik

Technische Universität Chemnitz-Zwickau

D-09107 Chemnitz, Germany

\begin{abstract}
:
A simple class of linear optimal control problems for parabolic equations with mixed control-state inequality constraints is investigated. The constraints are formulated pointwise in $L^{\infty}$. It is shown how to obtain associated Lagrange multipliers in $L^{p}$-spaces.
\end{abstract}

\section{INTRODUCTION}

We discuss the following linear optimal boundary control problem for the heat equation:

$$
\max \int_{\Omega} \alpha_{\Omega} y(T) d x+\int_{Q} \alpha_{Q} y d x d t+\int_{\Sigma} \alpha_{\Sigma} y d \sigma d t+\int_{\Sigma} \alpha_{u} u d \sigma d t
$$

subject to the state equation

$$
\begin{array}{rll}
y_{t}-\Delta y+d y=0 & \text { in } Q, \\
\partial_{\nu} y+b y=u & \text { on } \Sigma, \\
y(0)=0 & \text { in } \Omega,
\end{array}
$$

and to the mixed control-state constraints

$$
\begin{aligned}
& u(x, t) \leq c(x, t)+y(x, t), \\
& u(x, t) \geq 0,
\end{aligned}
$$

which are required a.e. on $\Sigma$. The heat equation is defined in a bounded domain $\Omega \subset \mathbb{R}^{N}, N \geq 2$, with sufficiently smooth boundary $\Gamma$. In the fixed 
time interval $(0, T)$ we put $Q:=\Omega \times(0, T)$, and $\Sigma:=\Gamma \times(0, T)$. Moreover, functions $\alpha_{\Omega} \in L^{\infty}(\Omega), d, \alpha_{Q} \in L^{\infty}(Q)$, and $c, b, \alpha_{\Sigma}, \alpha_{u} \in L^{\infty}(\Sigma)$ are given. The control function $u$ is assumed to be bounded and measurable. In this way, the feasible set of the control problem belongs to $L^{\infty}(\Sigma)$, and the mixed controlstate constraint $u \leq c+y$ must be regarded in the same space. Therefore, one might expect that an associated Lagrange multiplier has to be found in $L^{\infty}(\Sigma)^{*}$. In contrast to this, we shall verify the existence of at least one multiplier in $L^{\infty}(\Sigma)$. For linear programming problems in $L^{p}$-spaces with constraints of bottleneck type this surprising fact is known since long time, see for instance [1], [2], and the references cited therein. In this short note, we extend these early ideas to the parabolic boundary control problem defined above. Asscociated distributed control problems have been discussed extensively in our recent paper [3]. In the forthcoming paper [4], a class of nonlinear parabolic control problems with pointwise mixed control-state inequality constraints will be discussed on using these results.

We assume that $\Gamma$ is so smooth that a Green's function $G=G(x, \xi, t)$, $G: \bar{\Omega}^{2} \times \mathbb{R}_{+} \rightarrow \mathbb{R}$ exists, which satisfies the known estimate

$$
|G(x, \xi, t)| \leq k_{1} t^{-\frac{N}{2}} \exp \left(-k_{2} \frac{|x-\xi|^{2}}{t}\right)
$$

with positive real numbers $k_{1}, k_{2}$. Then the (weak) solution of (1.1) is given by

$$
y(x, t)=\int_{0}^{t} \int_{\Sigma} G(x, \xi, t-s) u(\xi, s) d \sigma(\xi) d s,
$$

where $d \sigma$ denotes the surface measure on $\Gamma$.

\section{COMPARISON PRINCIPLES FOR AN INTEGRAL EQUATION}

First we discuss the integral equation

$$
u(x, t)=f(x, t)+\int_{0}^{t} \int_{\Gamma} G(x, \xi, t-s) u(\xi, s) d \sigma(\xi) d s
$$

for given $f \in L^{\infty}(\Sigma)$. Introduce the integral operator $K$ :

$$
(K u)(x, t)=\int_{0}^{t} \int_{\Gamma} G(x, \xi, t-s) u(\xi, s) d \sigma(\xi) d s .
$$

$K$ is continuous in $L^{\infty}(\Sigma)$, also from $L^{p}(\Sigma)$ to $L^{\infty}(\Sigma)$ for $p>N+1$, see [5], p. 138 , Lemma 5.6.6. $K$ is also continuous from $L^{p}(\Sigma)$ to $C(\bar{Q})$. In this way, $y(\cdot, T)$ is well defined in $C(\bar{\Omega})$. Endow the space $L^{\infty}(\Sigma)$ with the equivalent norm $\|u\|_{\lambda}$,

$$
\|u\|_{\lambda}=\underset{(x, t) \in \Sigma}{\operatorname{vraimax}}\left|e^{-\lambda t} u(x, t)\right|,
$$

where $\lambda>0$. Then it is an easy and standard exercise to show the following result: 
Lemma $2.1 K$ is a contraction in $L^{\infty}(\Sigma)$, provided that $\lambda>\lambda_{o}$ is sufficiently large.

Corollary 2.1 For all $f \in L^{\infty}(\Sigma)$, the equation (2.1) has a unique solution $u \in L^{\infty}(\Sigma)$. The mapping $f \mapsto u$ is continuous in $L^{\infty}(\Sigma)$. If $G(x, \xi, t) \geq 0$ and $f(x, t) \geq 0$ a.e. in $\Sigma$, then $u(x, t) \geq 0$ a.e. in $\Sigma$.

Proof: We have

$$
u=(I-K)^{-1} f=\left(\sum_{n=0}^{\infty} K^{n}\right) f
$$

by well known results on Neumann series. Moreover, $\left\|(I-K)^{-1}\right\|_{\lambda} \leq 1 /(1-$ $\left.\|K\|_{\lambda}\right)$, where we have used for convenience the symbol $\|\cdot\|_{\lambda}$ to denote also the norm of the operator $K$ induced by $\|\cdot\|_{\lambda}$. The first part of the lemma follows from the equivalence of the norms $\|\cdot\|_{\lambda}$ and $\|\cdot\|_{L^{\infty}(\Sigma)}$. If $G$ is a nonnegative function, then $K$ is a nonnegative operator, that is $f \geq 0 \Rightarrow K f \geq 0$. The second result follows immediately from this and (2.4).

If $f_{1}, f_{2}$ are two bounded and measurable right hand sides for (2.1) and $G$ is nonnegative, then $u_{1} \geq u_{2}$ holds for the associated solutions. Now we verify these facts for the space $L^{p}(\Sigma)$. To see this, regard $K$ as an operator from $L^{p}(\Sigma)$ to $L^{\infty}(\Sigma)$. This smoothing property of $K$ is basic for the next result.

Corollary 2.2 (Comparison principle) Suppose $p>N+1$. Then the integral equation (2.1) has for each $f \in L^{p}(\Sigma)$ a unique solution $u \in L^{p}(\Sigma)$, and the mapping $f \mapsto u$ is continuous in $L^{p}(\Sigma)$. If $G(x, \xi, t) \geq 0, u_{i} \in L^{p}(\Sigma), i=1,2$, are solutions of (2.1) associated to $f_{i} \in L^{p}(\Sigma), i=1,2$, and $f_{1}(x, t) \geq f_{2}(x, t)$ holds a.e. in $\Sigma$, then $u_{1}(x, t) \geq u_{2}(x, t)$ a.e. in $\Sigma$.

Proof: Put $v:=u-f$, then (2.1) reads $v=K f+K v$. By the smoothing property of $K$, the right hand side $K f$ of this transformed equation is bounded and measurable. The last corollary implies that this equation admits a unique solution $v \in L^{\infty}(\Sigma)$ depending continuously on $K f$ and hence on $f$. Clearly, $u=f+v$ is a solution of $(2.1)$ in $L^{p}(\Sigma)$. The uniqueness of $u$ in $L^{p}(\Sigma)$ is and easy consequence, since the difference of two solutions solves the equation with right hand side zero, which belongs to $L^{\infty}(\Sigma)$. The comparison part of Corollary 2.2 follows from the arguments after Corollary 2.1 .

In a dual problem, which is defined later, the (formal) adjoint integral operator $K^{\top}$,

$$
\left(K^{\top} \mu\right)(x, t)=\int_{t}^{T} \int_{\Gamma} G(\xi, x, s-t) \mu(\xi, s) d \sigma(\xi) d s
$$

appears. $K^{\top}$ has the same properties as $K$. In particular, for $\lambda>\lambda_{o}$ it is a contraction in the norm $\|\cdot\|_{\lambda}$.

Lemma 2.2 For every function $a \in L^{\infty}(\Sigma)$, the equation

$$
\mu(x, t)=\max \left\{0, a(x, t)+\int_{t}^{T} \int_{\Gamma} G(\xi, x, s-t) \mu(\xi, s) d \sigma(\xi) d s\right\}
$$


has exactly one solution $\mu \in L^{\infty}(\Sigma)$.

The proof is an application of the contraction mapping principle. It can be applied, since the operator $(\Pi z)(x, t)=\max \{0, z(x, t)\}$ is Lipschitz continuous with Lipschitz constant one in $L^{\infty}(\Sigma)$, and $K^{\top}$ is a contraction.

\section{PRIMAL AND DUAL PROBLEM}

By inserting the integral representation (1.3) of the state $y$ in the objective functional of the control problem, we get after changing the order of integration

$\int_{\Omega} \alpha_{\Omega} y(T) d x+\int_{\Sigma} \alpha_{Q} y d x d t+\int_{\Sigma} \alpha_{\Sigma} y d \sigma d t+\int_{\Sigma} \alpha_{u} u d \sigma d t=\int_{\Sigma}(-a(x, t)) u(x, t) d \sigma d t$

with a certain function $a \in L^{\infty}(\Sigma)$ (a concrete expression for $a$ is given in section 5). In this way, the optimal control problem becomes a continuous linear programming problem with constraints of bottleneck type. This is our Primal Problem

$$
(\mathcal{P}) \begin{cases}\max \int_{\Sigma} a u d \sigma d t & \\ u(x, t) \leq[c+K u](x, t) & \text { a.e. in } \Sigma \\ u(x, t) \geq 0 & \text { a.e. in } \Sigma .\end{cases}
$$

Note that $(\mathcal{P})$ is defined in the space $L^{\infty}(\Sigma)$. From now on, we assume that $G(x, \xi, t-s) \geq 0$ and $p>N+1$. We define $u_{c}$ by the equation

$$
u_{c}=c+K u_{c} \text {. }
$$

According to Corollary 2.1, $u_{c}$ is bounded and measurable.

Theorem $3.1(\mathcal{P})$ has a solution $\bar{u}$ if and only if $u_{c} \geq 0$.

Proof: If $u_{c} \geq 0$, then the feasible set for $(\mathcal{P})$ is non-empty. All feasible elements $u \in L^{p}(\Sigma)$ satisfy $u \leq u_{c}$ by the comparison principle of Corollary 2.2, and we have $u_{c} \in L^{\infty}(\Sigma)$. Moreover, $u \geq 0$ follows from the constraints. This implies the $L^{\infty}$-boundedness of $u$ by $\left\|u_{c}\right\|_{L^{\infty}(\Sigma)}$. So the feasible set is bounded, closed, and convex in all (reflexive) $L^{p}$-spaces for $1+N<p<\infty$. The existence of $\bar{u} \in L^{p}(\Sigma)$ is an immediate conclusion. Of course $\bar{u}$ belongs to $L^{\infty}(\Sigma)$. On the other hand, if $u_{c}$ is not greater or equal than 0 , then the feasible set is empty by the comparison principle.

To introduce the dual problem to $(\mathcal{P})$, we extend the feasible set of $(\mathcal{P})$ from $L^{\infty}(\Sigma)$ to $L^{p}(\Sigma)(p>N+1)$. Any feasible solution $u$ of $(\mathcal{P})$ satisfies $0 \leq u \leq u_{c}$, where $u_{c}$ is defined in (3.1). Therefore, all feasible solutions of $L^{p}(\Sigma)$ belong automatically to $L^{\infty}(\Sigma)$, and the feasible set is not influenced by 
this extension to $L^{p}(\Sigma)$. From standard techniques to establish dual problems (see our discussion in section 6), in $L^{q}(\Sigma)$ we get the following Dual Problem:

$$
(\mathcal{D}) \begin{cases}\min \int_{\Sigma} c \mu d \sigma d t & \\ \mu(x, t) \geq\left[a+K^{\top} \mu\right](x, t) & \text { a.e. in } \Sigma \\ \mu(x, t) \geq 0 & \text { a.e. in } \Sigma\end{cases}
$$

where $\mu \in L^{q}(\Sigma), \frac{1}{p}+\frac{1}{q}=1$, and $K^{\top}$ is the adjoint integral operator defined by (2.5). The kernel of $K^{\top}$ satisfies the estimate (1.2), hence $K^{\top}: L^{p}(\Sigma) \rightarrow$ $L^{\infty}(\Sigma)$ holds for $p>N+1$, too. However, this is not true from $L^{q}(\Sigma)$ to $L^{\infty}(\Sigma)$. On the other hand, $K^{\top}$ represents the adjoint operator of $K: L^{p}(\Sigma) \rightarrow$ $L^{\infty}(\Sigma) \subset L^{p}(\Sigma)$. Therefore, $K^{\top}$ is continuous in $L^{q}(\Sigma) \sim L^{p}(\Sigma)^{*}$, too.

In view of $(1.2)$ and Lemma $2.1, K^{\top}$ is a contraction in $L^{\infty}(\Sigma)$, hence the equation

$$
\mu=\beta+K^{\top} \mu
$$

has a unique solution $\mu \in L^{\infty}(\Sigma)$ for each $\beta \in L^{\infty}(\Sigma)$. Moreover, we have uniqueness for (3.2) in $L^{q}(\Sigma)$. This can be shown by duality, since by Corollary (2.2) the range of $v-K v$ is $L^{p}(\Sigma)$. The next theorem contains the basic idea of this paper.

Theorem 3.2 If $c \geq 0$, then $(\mathcal{D})$ has at least one bounded and measurable optimal solution $\bar{\mu}$.

Proof: We know by Lemma 2.2 that equation (2.6)

$$
\mu(x, t)=\max \left\{0, a(x, t)+\left(K^{\top} u\right)(x, t)\right\}
$$

has in $L^{\infty}(\Sigma)$ exactly one solution $\bar{\mu}$. Let $\mu \in L^{q}(\Sigma)$ be any other feasible element for $(\mathcal{D})$, which is different from $\bar{\mu}$. Then $\mu \geq a+K^{\top} \mu$ a.e. in $\Sigma$ and, of course, $\mu \geq 0$. Next, we construct a sequence $\mu_{1} \geq \mu_{2} \geq \cdots$ as follows : $\mu_{1}=\mu \in L^{q}(\Sigma)$ and $\mu_{2}=\max \left\{0, a+K^{\top} \mu_{1}\right\}$. A simple discussion yields $\mu_{2} \leq \mu_{1}$ a.e. in $\Sigma$. Then by positivity, $K^{\top} \mu_{2} \leq K^{\top} \mu_{1}$, and we get

$$
\mu_{2} \geq a+K^{\top} \mu_{1} \geq a+K^{\top} \mu_{2} \text { a.e. on } \Sigma \text {. }
$$

So $\mu_{2}$ is feasible and $\mu_{2} \leq \mu_{1}$ on $\Sigma$. Repeating this process, one constructs a non-increasing feasible sequence $\left\{\mu_{n}\right\}$ which has to be pointwise convergent towards some $\tilde{\mu} \geq 0$, that is

$$
\lim _{n \rightarrow+\infty} \mu_{n}(x, t)=\tilde{\mu}(x, t) \text { a.e. on } \Sigma \text {. }
$$

An application of the Lebesgue dominated convergence theorem yields that $\mu_{n}$ tends to $\tilde{\mu}$ in $L^{q}(\Sigma)$. Passing to the limit in $\mu_{n}=\max \left\{0, a+K^{\top} \mu_{n-1}\right\}$ gives in $L^{q}(\Sigma)$

$$
\tilde{\mu}=\max \left\{0, a+K^{\top} \tilde{\mu}\right\} .
$$


Finally, by simple arguments, we are able to conclude $\tilde{\mu} \in L^{\infty}(\Sigma)$. By uniqueness in $L^{\infty}(\Sigma)$ we have $\tilde{\mu}=\bar{\mu}$; moreover $\mu \geq \tilde{\mu}$. Therefore $\mu \geq \bar{\mu}$ holds for all feasible solutions and, since $c \geq 0$,

$$
\int_{\Sigma} c(x, t) \mu(x, t) d \sigma d t \geq \int_{\Sigma} c(x, t) \bar{\mu}(x, t) d \sigma d t .
$$

\section{THE DUALITY RELATION}

The discussion of the duality between $(\mathcal{P})$ and $(\mathcal{D})$ is not yet complete. We have only shown that under certain assumptions the dual problem admits a solution $\bar{\mu}$. To make sure that $\bar{\mu}$ is a Lagrange multiplier associated to a solution of $(\mathcal{P})$, we need additionally the strong duality relation, that is the equality of primal and dual optimal value. To this aim, we briefly sketch some main ideas of duality for linear programs.

Let $X=L^{p}(\Sigma)$ with its natural partial ordering $\geq$, and define $A:=I-K$ having the adjoint operator $A^{\prime}$. By $\langle\cdot, \cdot\rangle$ we denote the pairing between $X$ and its dual space $X^{\prime}=L^{q}(\Sigma)$. Then the primal problem is

$$
\begin{aligned}
\max & \langle a, x\rangle \\
A x & \leq c \\
x & \geq 0 .
\end{aligned}
$$

On using the Lagrange function $\mathcal{L}(x, \mu):=\langle a, x\rangle+\langle\mu, c-A x\rangle$, the primal problem can be written in sup-inf form. Reversing the order of supremum and infimum we arrive at the dual problem

$$
\begin{aligned}
\min _{A^{\prime}} & \langle\mu, c\rangle \\
\mu & \geq a \\
\mu & \geq 0 .
\end{aligned}
$$

Let $\bar{x}$ be optimal for $(\mathcal{P})$. Then $\bar{\mu}$ is an associated Lagrange multiplier if and only if the pair $(\bar{x}, \bar{\mu})$ is a saddle point of $\mathcal{L}$, i.e. $\mathcal{L}(x, \bar{\mu}) \leq \mathcal{L}(\bar{x}, \bar{\mu}) \leq \mathcal{L}(\bar{x}, \mu)$ for all $x \geq 0, \mu \geq 0$. A necessary and sufficient condition for $(\bar{x}, \bar{\mu})$ to be a saddle point is that $\bar{x}$ solves $(\mathcal{P}), \bar{\mu}$ solves $(\mathcal{D})$, and the strong duality relation $v=v^{\prime}$ holds true. Let us define by $P(c)$ and $D(a)$ the feasible sets of $(\mathcal{P})$ and $(\mathcal{D})$, respectively, i.e.

$$
P(c)=\{x \in X \mid x \geq 0, A x \leq c\}, \quad D(a)=\left\{\mu \in X^{\prime} \mid \mu \geq 0, A^{\prime} \mu \geq a\right\} .
$$

It is easy to verify that the weak duality relation

$$
\sup _{x \in P(c)}\langle a, x\rangle \leq \inf _{\mu \in D(a)}\langle\mu, c\rangle
$$

holds always true. To show the strong duality relation, we need the convex cone

$$
K(A):=\{(\alpha, d) \in \mathbb{R} \times X \mid \exists x \geq 0: A x \leq d,\langle a, x\rangle \geq \alpha\} .
$$


Theorem 4.1 If $K(A)$ is closed and $(\mathcal{P})$ admits at least one solution, then the strong duality relation holds, that is

$$
\max _{x \in P(c)}\langle a, x\rangle=\inf _{\mu \in D(a)}\langle\mu, c\rangle
$$

This is a standard result of linear programming theory. We should mention that (4.2) implies the complementary slackness conditions

$$
\langle A \bar{x}-c, \bar{\mu}\rangle=\left\langle A^{\prime} \bar{\mu}-a, \bar{x}\right\rangle=0 .
$$

It turns out that the assumptions of the theorem are fulfilled for our problem:

Lemma 4.1 $K(A)$ is closed for $(\mathcal{P})$.

We know from the proof of the Theorem 3.1 that the norm of $u \in P(c)$ is bounded by the norm of $u_{c}$, which is bounded by Corollary 2.2. Therefore, this Lemma is easy to prove.

\section{NECESSARY OPTIMALITY CONDITIONS}

Finally, we prove that the solution $\bar{\mu}$ of $(\mathcal{D})$ is a Lagrange multiplier for the state-constraint of the parabolic control problem. By definition, $\bar{\mu}$ is an associated Lagrange multiplier, if $\bar{u}$, together with $\bar{\mu}$ and an adjoint state $\bar{p}$, satisfies the first order necessary optimality conditions: They consist of the adjoint equation

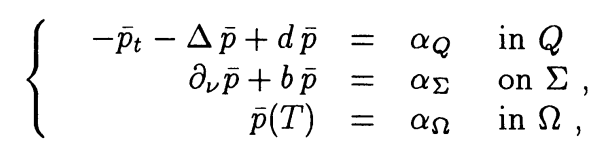

the variational inequality

$$
\int_{\Sigma}\left(\alpha_{u}+\bar{p}+\bar{\mu}\right)(u-\bar{u}) d \sigma d t \geq 0 \quad \forall u \geq 0
$$

the complementary slackness condition

$$
(\bar{u}-c-\bar{y}) \bar{\mu}=0 \quad \text { a.e. in } \Sigma,
$$

and the nonnegativity condition $\bar{\mu}(x, t) \geq 0$, which must be satisfied a.e. on $\Sigma$.

To verify these conditions, we introduce some auxiliary functions: Put $a_{\Omega}=$ $-\alpha_{\Omega}, a_{\Sigma}=-\alpha_{\Sigma}, a_{Q}=-\alpha_{Q}, a_{u}=-\alpha_{u}$. Moreover, we define $\Psi$ and $\varphi$ by

$$
\begin{aligned}
-\Psi_{t}-\Delta \Psi+d \Psi & =a_{Q} & -\varphi_{t}-\Delta \varphi+d \varphi & =0 \\
\partial_{\nu} \Psi+b \Psi & =a_{\Sigma} & \partial_{\nu} \varphi+b \varphi & =\bar{\mu} \\
\Psi(T) & =a_{\Omega} & \varphi(T) & =0
\end{aligned} .
$$


The function $\varphi$ plays the role of the dual state. It holds $K^{\top} \bar{\mu}=\varphi$, hence the constraints of $(\mathcal{D})$ admit the form $\mu \geq a+\varphi$. Moreover, (4.3) yields the complementary slackness condition for the dual problem,

$$
(-a-\varphi+\bar{\mu}) \bar{u}=0 \quad \text { a.e. on } \Sigma \text {. }
$$

$\Psi$ was defined to satisfy $a=\Psi+a_{u}=\Psi-\alpha_{u}$.

It is clear that we have to put $\bar{p}:=-(\Psi+\varphi)$. Then $p$ solves (5.1). Moreover, the complementary slackness condition (5.3) is satisfied by (4.3). Finally, the variational inequality follows from

$$
\begin{aligned}
& \int_{\Sigma}\left(\alpha_{u}+\bar{p}+\bar{\mu}\right)(u-\bar{u})=\int_{\Sigma}\left(-a_{u}+\bar{p}+\bar{\mu}\right)(u-\bar{u})=\int_{\Sigma}(\Psi-a+\bar{p}+\bar{\mu})(u-\bar{u}) \\
& =\int_{\Sigma}(-a-\varphi+\bar{\mu})(u-\bar{u})=\int_{\Sigma}(-a-\varphi+\bar{\mu}) u-\int_{\Sigma}(-a-\varphi+\bar{\mu}) \bar{u} \geq 0 .
\end{aligned}
$$

In the last estimate, the relations $-a-\varphi+\mu \geq 0$ and (5.4) were used. The optimality conditions are verified. Altogether, we have found our final result:

Theorem 5.1 The dual problem (D) has at least one bounded and measurable solution $\bar{\mu}$. Let $\bar{u}$ be optimal for the linear parabolic boundary control problem. Then $\bar{\mu}$ is a Lagrange multiplier associated with the mixed control-state constraint $u \leq c+y$.

\section{References}

[1] E.J. Anderson, A review of duality theory for linear programming over topological vector spaces, Journal of Mathematical Analysis and Applications 97, 380-392 (1983).

[2] E.J. Anderson and P. Nash, Linear programming in infinite-dimensional spaces, J. Wiley \& Sons, Chichester etc. 1987.

[3] M. Bergounioux and F. Tröltzsch, Optimal control of linear bottleneck problems, ESAIM: Control, Optimisation and Calculus of Variations, Vol. $3, \mathrm{n}^{\circ}$ 5\&6, pp.235-250 (1998).

[4] M. Bergounioux and F. Tröltzsch, Optimal control of semilinear parabolic equations with state-constraints of bottleneck type, Faculty of Math., TU Chemnitz, Preprint 98-19, to appear.

[5] F. Tröltzsch, Optimality conditions for parabolic control problems and applications, Teubner Texte zur Mathematik, Leipzig (1984). 